\title{
Respuesta no lineal de estructuras con muros de concreto reforzado
}

\section{Non-linear response of reinforced concrete wall structures}

\author{
DOI: https://doi.org/10.17981/ingecuc.14.2.2018.05
}

Artículo de investigación científica. Fecha de recepción: 15/02/2018. Fecha de aceptación: 29/06/2018.

\author{
Alberto Vásquez Martínez \\ Universidad Nacional Autónoma de México. Ciudad de México (México) \\ ing.albertovm@gmail.com \\ Romel Jesús Gallardo Amaya \\ Universidad Francisco de Paula Santander. Ocaña (Colombia) \\ rjgallardoa@ufpso.edu.co
}

Para citar este artículo:

A. Vásquez Martínez y R. J. Gallardo Amaya, "Respuesta no lineal de estructuras con muros de concreto reforzado," INGE CUC, vol. 14, no. 2, pp. 55-61, 2018. DOI: http://doi.org/10.17981/ingecuc.14.2.2018.05

\section{Resumen}

Introducción-Muchas edificaciones compuestas por marcos (vigas-columnas) tienen la necesidad de recurrir a muros de concreto reforzado para proporcionar una mayor rigidez lateral. El comportamiento no lineal de sistemas con muros de concreto reforzado es incierto y, por lo tanto, se requiere conocer de manera adecuada la respuesta estructural de estos ante diferentes excitaciones sísmicas por medio de criterios adecuados de modelación.

Objetivo- Este estudio tiene como finalidad encontrar una metodología adecuada de modelación para muros de concreto reforzado considerando discretizaciones tipo armadura. Para validación se utiliza uno de los especímenes presentados en un informe experimental, en donde se reportan pruebas a distintos muros sometidos a cargas cíclicas de desplazamientos que caracterizan prototipos reales.

Metodología- Se utiliza como modelo de calibración los resultados de ensayo del espécimen experimental No. 6, presentados en el reporte No. UBC/EERC-79/20 realizado en la universidad de California, Berkeley. El espécimen es sometido a una carga lateral cíclica reversible y cargas concentradas debido a los pisos superiores, estas cargas son utilizadas como excitación para el modelo analítico. Las características geométricas y propiedades mecánicas son tomadas del informe para la modelación analítica.

Resultados- Con la metodología anterior es posible modelar cualquier muro de concreto reforzado bidimensional. Los resultados obtenidos en términos del cortante basal y desplazamiento en la azotea son comparados con los mostrados en el informe experimental. La curva de histéresis global $\left(\delta_{3} v s V_{b}\right)$ es obtenida sometiendo al modelo analítico a una carga pseudoestática reversible y considerando los parámetros no lineales de cada uno de los materiales.

Conclusiones- Los resultados obtenidos con el modelo analítico discretizado en elementos armadura presentan una gran aproximación con los mostrados en el estudio experimental utilizado como comparación, por lo cual, este criterio es posible utilizarlo para modelar cualquier tipo de muro de concreto reforzado en el espacio bidimensional e incluso tridimensional.

Palabras clave- Carga cíclica; comportamiento histerético; cortante; flexión; modelo armadura; muro estructural; no lineal

\section{Abstract}

Introduction- Many buildings composed of frames (beams-columns) must resort to reinforced concrete walls to provide greater lateral stiffness. The nonlinear behavior of systems with reinforced concrete walls is uncertain and, therefore, it is necessary to know adequately the structural response of these to different seismic excitations by means of suitable modeling criteria.

Objective- This study aims to find an adequate modeling methodology for reinforced concrete walls considering frame type discretizations. For validation, one of the specimens presented in an experimental report is used, where tests are reported to different walls subjected to cyclic displacement loads that characterize real prototypes.

Methodology - The experimental results of experimental specimen No. 6, presented in the report No. UBC/EERC-79/20 conducted at the University of California, Berkeley, are used as a calibration model. The specimen is subjected to a reversible cyclic lateral load and concentrated loads due to the upper floors, these loads are also used as excitation loads for the analytical model. The geometric characteristics and mechanical properties are taken from the report for analytical modeling.

Results- With the previous methodology, it is possible to model any two-dimensional reinforced concrete wall. The results obtained in terms of basal shear and displacement on the roof are compared with those shown in the experimental report. The global hysteresis curve $\left(\delta_{3}\right.$ vs $\left.V_{b}\right)$,) is obtained by subjecting the analytical model to a reversible pseudo-static load and considering the non-linear parameters of each of the materials.

Conclusions- The results obtained with the analytic model discretized in frame elements present a great approximation with those shown in the experimental study used as a comparison, therefore, this criterion can be used to model any type of reinforced concrete wall in two-dimensional space and even threedimensional.

Keywords-Cyclic load; hysteretic behavior; shearing; bending; frame-type model; structural wall; non-linear 


\section{INTRODUCCIÓN}

Las estructuras de concreto reforzado de mediana a gran altura, ante un evento sísmico de intensidad elevada, pueden presentar una respuesta sísmica no lineal [1][2], la cual conduce a daños estructurales debido al incremento de los desplazamientos, elementos mecánicos y concentraciones de esfuerzos en las articulaciones plásticas. La mayoría de los edificios de concreto reforzado sometidos a dichos eventos sísmicos requieren de elementos estructurales que disminuyan los desplazamientos excesivos y que aporten mayor rigidez estructural, por lo anterior, es frecuente utilizar la combinación de columnas, vigas y muros de concreto reforzado [3], formando estructuras muy rígidas que disminuyen las deformaciones y distorsiones de entrepiso [4].

Existen muchas técnicas de modelación con muros de concreto reforzado, tales como: elementos finitos, columna ancha, secciones tipo fibra, armadura equivalente [5], entre otros; sin embargo, los estudios sobre modelación de muros de concreto reforzado, tomando en cuenta los efectos no lineales e interacción de flexión-cortante, son escasos [6]. Este estudio se basa en la metodología desarrollada en [7], el cual propone una modelación tipo armadura que considera intrínsecamente la interacción flexión-cortante y, adicionalmente, la deformación biaxial debido a los efectos de compresión en la componente diagonal de la armadura [9][10].

Para fines de comparación de resultados y validación de la metodología desarrollada en [7][8], se utilizan los resultados del informe experimental mostrado en [11]. En ese informe se realizan pruebas experimentales en diversos especímenes de muros de concreto reforzado que se someten a distintas cargas pseudoestáticas. Estos especímenes tienen diferentes propiedades mecánicas en sus materiales y son idealizaciones que caracterizan prototipos de edificios de 7 y 10 niveles. De [11] se toman los resultados de la curva de histéresis del espécimen no. 6 en términos de desplazamiento en la azotea vs fuerza cortante en la base; estos resultados son comparados con los obtenidos de la modelación analítica desarrollada de acuerdo con [7].

\section{Metodología}

Para realizar los análisis no lineales de estructuras con muros de concreto reforzado, es necesario determinar la respuesta cíclica no lineal de dichos muros ante excitaciones sísmicas. Lo anterior, se logra utilizando un modelo no lineal cíclico de armadura propuesto por [7]. Este modelo caracteriza al muro de concreto reforzado como una armadura formada por elementos verticales, horizontales y diagonales que toman en cuenta intrínsecamente la interacción flexión-cortante y representan la degradación de rigidez y resistencia [12]. La interacción flexión-cortante puede afectar significativamente el comportamiento cíclico no lineal de los muros de concreto reforzado en términos de resistencia, rigidez y capacidad de deformación del sistema estructural. Para demostrar que los resultados que se obtendrían en sistemas estructurales con muros de concreto reforzados son adecuados, se realizó una calibración del modelo propuesto en [7]; para esto, se utilizan los resultados experimentales presentados en [11]. Se toman los resultados experimentales obtenidos en el espécimen 6 que consiste en un muro de concreto reforzado sujeto a carga cíclica reversible. Este espécimen es representado experimentalmente por 3 niveles y caracteriza un prototipo de un edificio de 7 niveles. Lo anterior se utiliza con la finalidad de comparar los resultados experimentales con los obtenidos en un modelo analítico.

\section{A. Espécimen experimental}

Este estudio se centra en la calibración de un modelo analítico con las características geométricas y propiedades mecánicas del espécimen 6 , tomado del informe experimental de [11]. Las características geométricas en altura y planta, espesores del muro de concreto, así como el calibre y separación de los aceros estructurales se muestran en la Fig. 1. Las propiedades mecánicas de los diversos materiales utilizados para la declaración de los modelos de comportamiento histerético presentados en incisos posteriores, tales como: resistencia a compresión del concreto, resistencia a la fluencia del acero, resistencia última del acero, módulos de elasticidad del acero y concreto, deformaciones iniciales y últimas del acero, entre otras propiedades, son tomadas de [11]. El espécimen mostrado en la Fig. 1 consta de 3 niveles con una viga rígida en la parte superior con actuadores de carga en donde se concentran cargas debidas al peso de los pisos superiores, que representan los prototipos estudiados en el informe de [11]. El panel de concreto tiene un espesor de 0,114 m con losas diafragmas en cada nivel.

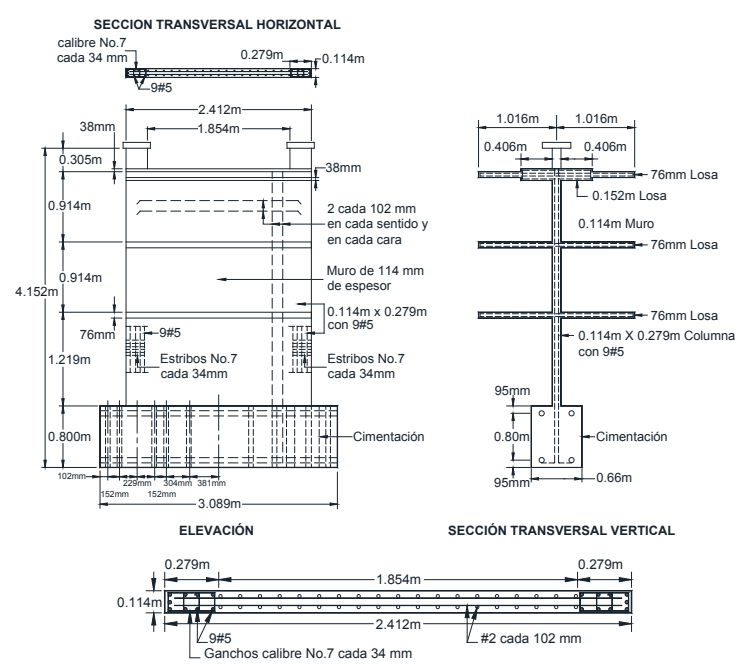

Fig. 1. Prototipo que caracteriza un edificio de 7 niveles. Fuente: Adaptada de [10]. 


\section{B. Modelo analítico de calibración}

El modelo armadura que caracteriza al espécimen 6 se presenta en la Fig. 2 [13], la cual muestra los elementos horizontales, verticales y diagonales que forman la armadura. Los elementos horizontales y verticales se representan por áreas de concreto y acero. Las áreas de concreto y acero se determinan de acuerdo con los anchos y longitudes tributarias que les correspondan a las subdivisiones propuestas en el panel de concreto y columnas de borde, respectivamente. Los elementos diagonales de la armadura se representan solamente por el área de concreto, y esta se determina mediante un ancho efectivo $b_{\text {ef }}$ multiplicado por el espesor del panel de concreto. Para el modelo bidimensional analítico no lineal sujeto a una carga pseudoestática reversible, se empleó el programa libre Opensees [14].

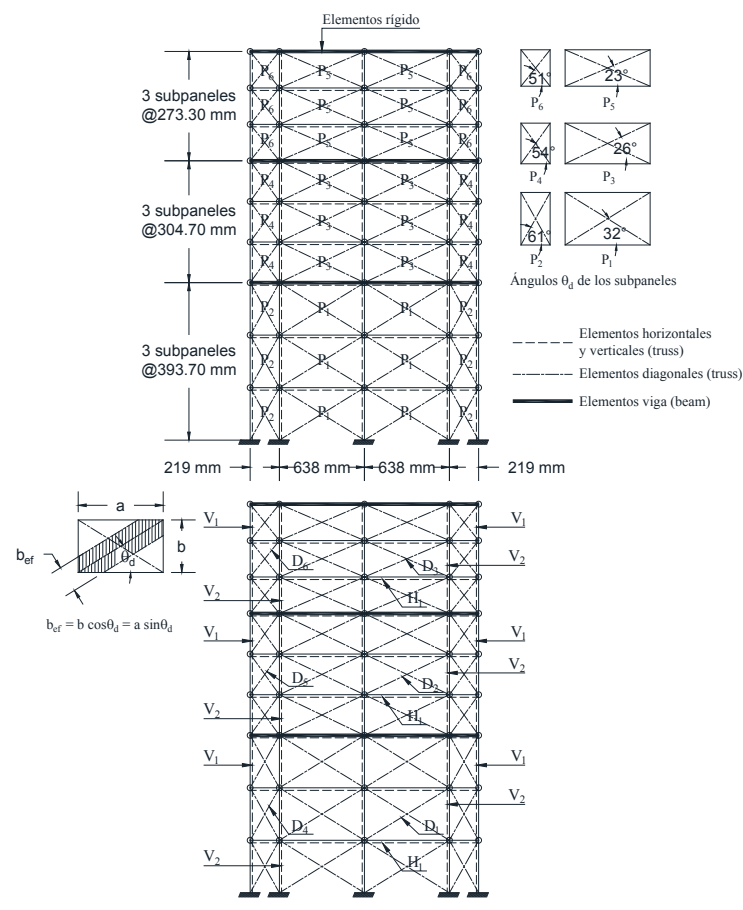

Fig. 2. Configuración del espécimen 6 de [11] como modelo armadura de acuerdo con [7] Fuente: Autores.

Los subpaneles P2, P4 y P6 mostrados en la Fig. 2 representan las columnas de borde; mientras que, los subpaneles P1, P3 y P5 representan el alma del muro de concreto reforzado. Los criterios para descretizar el muro de concreto reforzado se obtienen de [7].

Las áreas de concreto y acero para los elementos verticales, horizontales y diagonales se muestran en la tabla 1.
Tabla 1. Áreas de CONCReto y aCero de los ELEMENTOS QUE FORMAN LA ARMADURA.

\begin{tabular}{|c|c|c|}
\hline Elemento & Área de concreto $\left(\mathrm{mm}^{2}\right)$ & Área de acero $\left(\mathrm{mm}^{2}\right)$ \\
\hline $\mathrm{V}_{1}$ & 15903 & 989,7 \\
\hline $\mathrm{V}_{2}$ & 15903 & 791,7 \\
\hline $\mathrm{V}_{3}$ & 105678 & 601,7 \\
\hline $\mathrm{H}_{1}$ & 36923,3 & 2276 \\
\hline $\mathrm{D}_{1}$ & 38061,9 & \\
\hline $\mathrm{D}_{2}$ & 21759,1 & \\
\hline $\mathrm{D}_{3}$ & 31220,3 & \\
\hline $\mathrm{D}_{4}$ & 20417,2 & \\
\hline $\mathrm{D}_{5}$ & 28679,4 & \\
\hline $\mathrm{D}_{6}$ & 19607,2 & \\
\hline
\end{tabular}

Fuente: Autores.

\section{Modelo de comportamiento no lineal del concreto para los elementos verticales y horizontales}

El modelo de comportamiento utilizado para el concreto no confinado o confinado es el "ConcretewBeta” (Fig. 3), que considera explícitamente el efecto de deformación normal para el comportamiento del concreto en compresión [15]. La envolvente esfuerzo-deformación del concreto confinado o no confinado se basa en los estudios de [16]. Se considera que la degradación de resistencia en tensión es trilineal. Los parámetros en la envolvente de compresión que definen el modelo de comportamiento ConcretewBeta se tomaron del informe experimental de [11]. Algunos parámetros no encontrados en [11] se toman del estudio detallado en [7]. Se considera concreto no confinado en el muro, mientras que, en las columnas concreto confinado. Se utiliza el comando element truss en el programa Opensees, y los parámetros que requiere este comando se toman de [11].

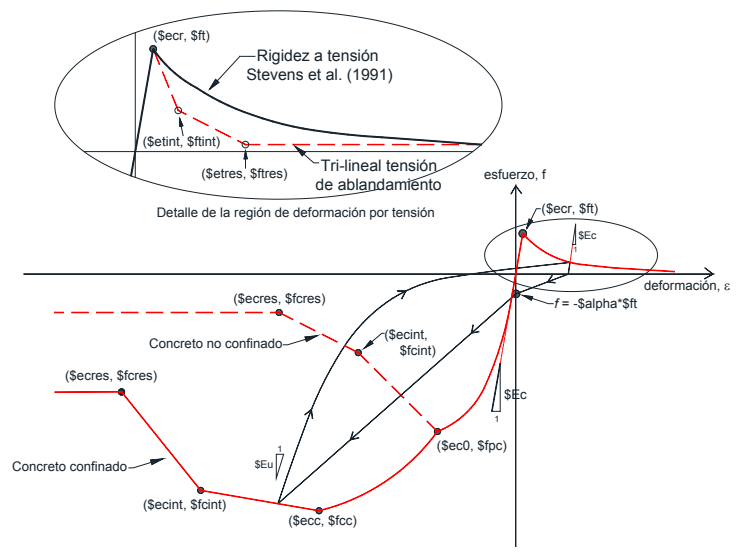

Fig. 3. Modelo de comportamiento del material ConcretewBeta. Fuente: Adaptada de [14]. 


\section{Modelo de comportamiento del concreto para los elementos diagonales}

El modelo empleado para los elementos diagonales es similar al de los elementos verticales y horizontales (Fig. 4 y 5), con la diferencia de que no se tiene resistencia a la tensión del concreto y, además, que se considera la deformación biaxial en el comportamiento a compresión del concreto, tal como se explica en [17]. Los parámetros que se muestran en la Fig. 5 se toman de [7]. En el programa Opensees se utiliza el comando element truss 2 para representar los elementos diagonales. La diagonal 3-4 mostrada en la Fig. 4 representa un elemento de medición de la deformación normal $\varepsilon_{n}$.

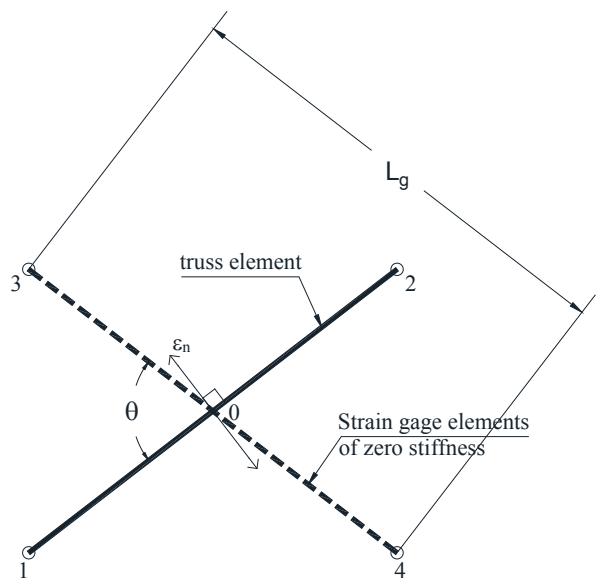

Fig. 4. Elemento armadura que toma en cuenta los efectos biaxiales en el comportamiento esfuerzo-deformación del concreto a compresión. Fuente: Tomada de [7]

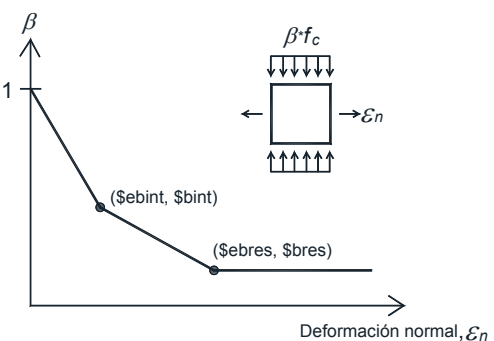

Fig. 5. Relación entre el factor de reducción $\beta$ del esfuerzo a compresión y el esfuerzo normal $\varepsilon_{n}$. Fuente: Tomada de [7].

\section{E. Modelo de comportamiento del acero}

Para el comportamiento del acero de refuerzo se utiliza el modelo "Steel02" [18][19]. Los principales parámetros de los que depende este modelo son: el esfuerzo de fluencia $f_{y}$; módulo de elasticidad $E$; un parámetro $R$ que controla la transición de la rama elástica a la plástica (valores recomendados entre 10 y 20); $c_{1}$ y $c_{2}$ que son coeficientes que modifican el parámetro $R$ con ciclos de carga (se recomiendan valores de 0,925 y 0,15$) ; a_{1}$ y $a_{2}$ son parámetros determinados experimentalmente que toman en cuenta el endurecimiento isotrópico en la zona de compresión; $a_{3}$ y $a_{4}$ son parámetros experimentales que toman en cuenta el endurecimiento isotrópico en la zona de tensión. Para este estudio no se toma en cuenta el endurecimiento isotrópico por deformación y, por lo tanto, los parámetros $a_{1}, a_{2}, a_{3}$ y $a_{4}$ no son asignados dentro del modelo Steel02. Los parámetros como $f_{y}$ y $E$ de cada varilla utilizada en el muro y columnas de concreto se toman del informe experimental de [11].
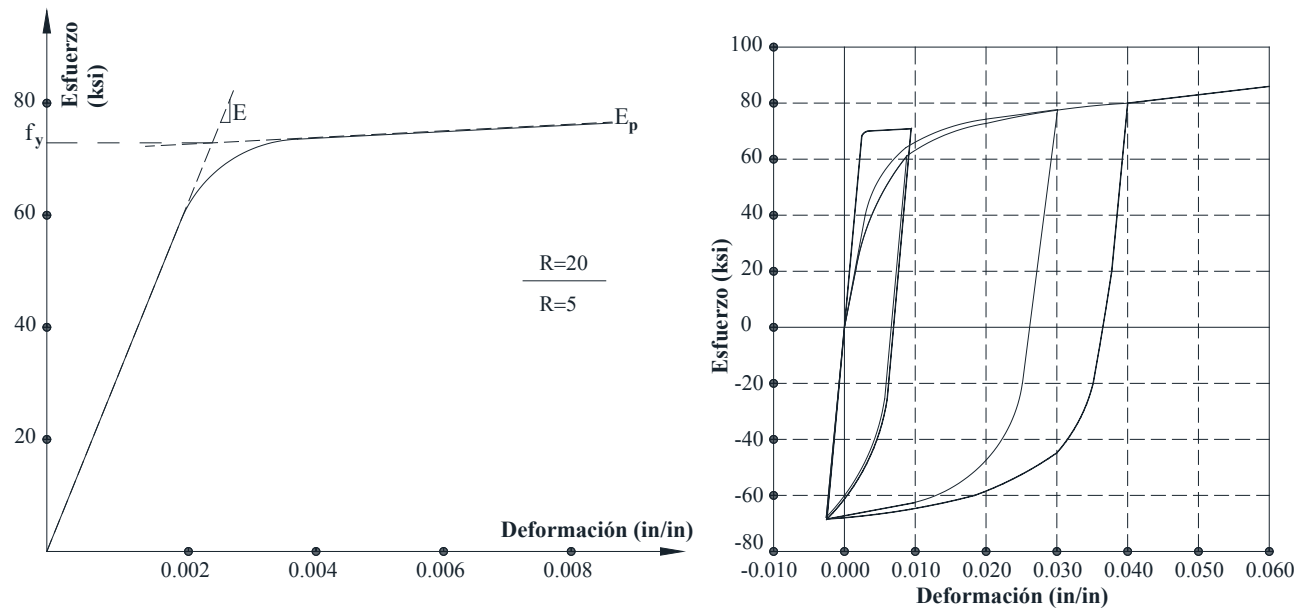

Fig. 6. Modelo de comportamiento del material Steel02.

Fuente: Adaptada de [14]. 


\section{F. Excitación pseudoestática}

Como excitación lateral se utilizan unas secuencias pre-establecidas de cargas, por ejemplo, las utilizadas en el espécimen 6 del informe experimental mostrado en [11]. Esta carga cíclica reversible actúa en el centro de masa del ultimo nivel del marco bidimensional utilizado como modelo de calibración. La historia cíclica de desplazamientos que actúan como carga lateral reversible se muestra en la Fig. 7 [20]. En la Fig. 8 se observa la historia de fuerzas cortantes en la base tomadas de [11].

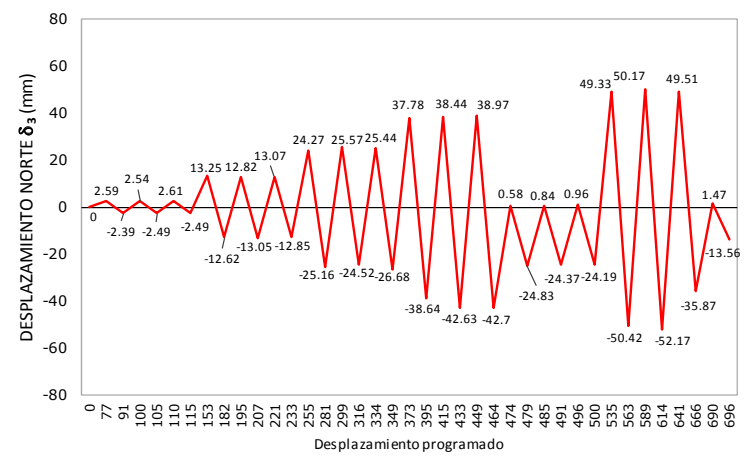

Fig. 7. Historia de desplazamiento en el nivel 3 del espécimen 6 y modelo analítico. Fuente: Adaptada de [11].

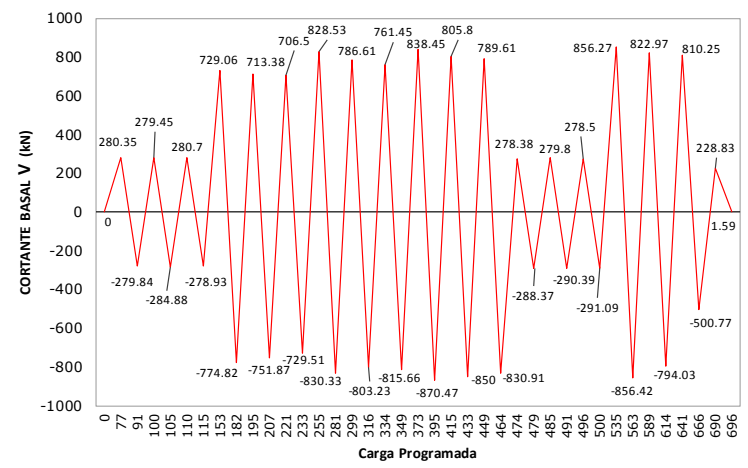

Fig. 8. Historia de fuerza cortante en la base de acuerdo con [11]. Fuente: Adaptada de [11].

\section{G. Análisis no lineal}

El análisis pseudoestático no lineal del modelo analítico de calibración se realizó con el programa Opensees [14]. Los elementos estructurales que conforman el muro de concreto reforzado, tales como: elementos verticales, horizontales y diagonales, siguen el comportamiento no lineal que se mencionan en los incisos C, D y E.

Como se había mencionado, el modelo analítico de 3 niveles es representativo de un prototipo de un edificio de 7 niveles, de acuerdo con [11], y, por lo tanto, adicionalmente se considera la acción de fuerzas gravitacionales concentradas en los nudos de la esquina del último nivel; dichas fuerzas son generadas a partir de los pesos que producen los pisos superiores.
La Fig. 9 muestra esquemáticamente las fuerzas gravitacionales que se consideran para el análisis no lineal.

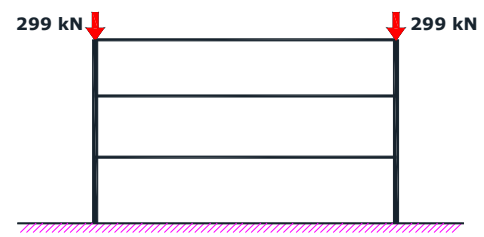

Fig. 9. Cargas gravitacionales consideradas en el modelo analítico. Fuente: Adaptada de [11].

\section{III.RESULTADOS}

\section{A. Resultados del análisis no lineal}

La respuesta global fue medida en el centro de masa del último nivel (nivel 3) y se obtuvo en términos de la historia de desplazamientos " $\delta_{3}$ " en la azotea, en las direcciones de análisis X; además, se determinó la historia de cortantes en la base " $V_{b}$ " correspondiente a la dirección " $\delta_{3}$ ". Con lo anterior, se puede representar la curva cortante basal-desplazamiento de la azotea $\left(V_{b}-\delta_{3}\right)$, ver Fig. 10 .

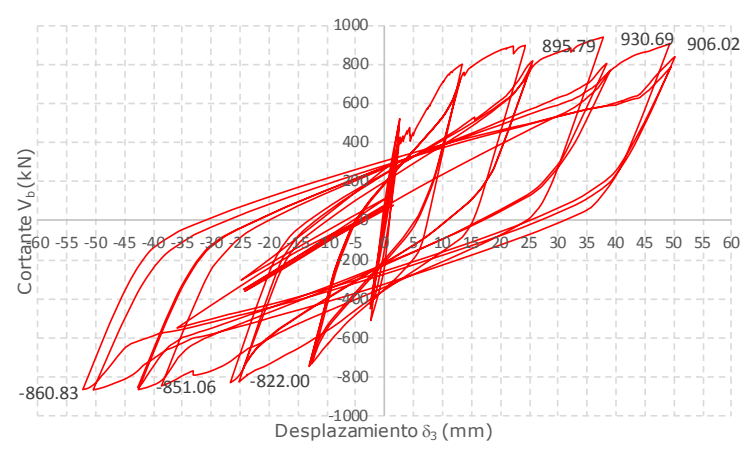

Fig. 10. Curva de histéresis analítica. Fuente: Autores.

De [10] se obtiene la curva de histéresis global objetivo de desplazamiento en la azotea (nivel 3) vs la fuerza cortante en la base. Esa curva se compara con los resultados obtenidos y mostrados en la Fig. 11 del modelo analítico.

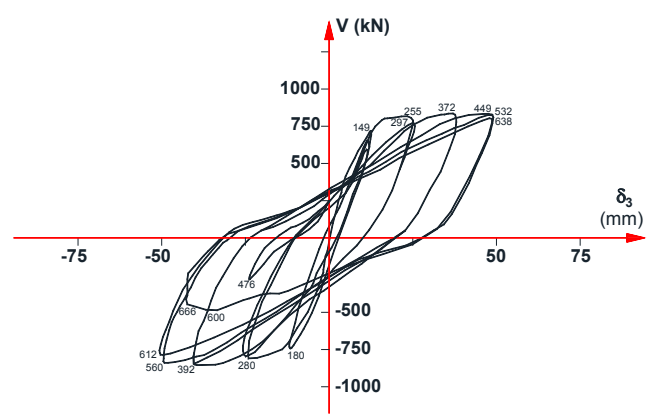

Fig. 11. Curva de histéresis experimental. Fuente: Adaptada de [11]. 


\section{B. Comparativa de curvas de histéresis}

Después de realizar diferentes calibraciones, se presenta, en la Fig. 10, la curva de histéresis global de cortante en la base " $V_{b}$ " vs el desplazamiento " $\delta_{3}$ " en el nivel 3. Los resultados obtenidos con el modelo analítico muestran la gran aproximación con los resultados experimentales obtenidos en [11], que se muestran en la Fig. 11.

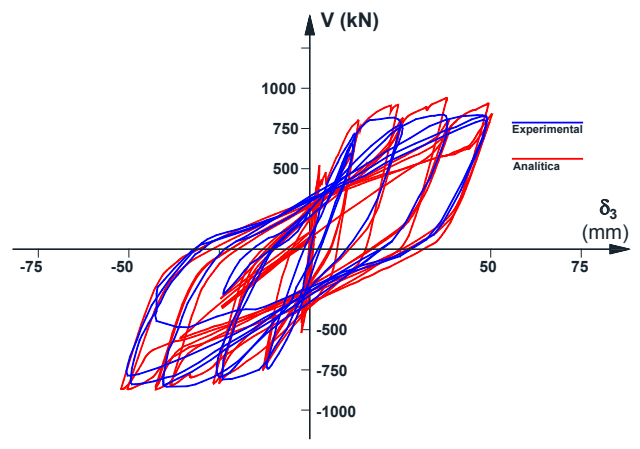

Fig. 12. Comparativas entre las curvas de histéresis analítica y experimental. Fuente: Autores

En la Fig. 12, se observa una buena aproximación entre los valores obtenidos analíticamente y los experimentales. De igual manera, es posible observar que en el número de lazos es prácticamente similar y que el ancho de la curva coincide en el punto de intercepción del eje vertical de la fuerza cortante basal; además que la pendiente inicial de ambas curvas es semejante. La no exactitud de los valores de los picos, sobre todo en términos de fuerza cortante, se debe particularmente a que los resultados experimentales fueron obtenidos en condiciones reales de laboratorio, mientras que, los resultados analíticos son basados en aspectos teóricos ya antes mencionados.

\section{Conclusiones}

Se obtuvo una metodología para estimar con buena aproximación los efectos no lineales en estructuras bidimensionales con muros de concreto reforzado (MCR) utilizando el criterio desarrollado por [7]. Con esta metodología es posible discretizar cualquier muro de concreto reforzado en el plano, considerando la interacción flexión-cortante y deformación normal del concreto debido a los efectos de compresión en la diagonal de la armadura.

La metodología antes expuesta es aplicable a estructuras bidimensionales; por lo tanto, en estudios futuros se puede investigar la posibilidad de implementar esta metodología en estructuras tridimensionales con muros de concreto reforzado, considerando a las columnas como un elemento barra discretizado en fibras. En modelos tridimensionales es posible considerar la contribución de las losas de entrepiso como elementos tipo armadura, mientras que, las vigas y columnas pueden modelarse como elementos barra por medio de secciones discretizadas en fibras e integradas a lo largo de los elementos mediante funciones de forma de los desplazamientos; para esto se utiliza el modelo de plasticidad de [21]. Lo anterior es un gran esfuerzo para poder conocer la compatibilidad de deformaciones en los nudos donde concurren elementos armadura restringidas rotacionalmente con elementos liberados en todas sus direcciones.

En este estudio se utilizó como excitación una carga pseudoestática; sin embargo, pueden realizarse calibraciones con una excitación dinámica en la base con la finalidad de observar las variaciones que existen en los resultados de la historia de desplazamientos en el tiempo, en comparación con los obtenidos de un análisis pseudoestático.

Es de interés mencionar lo complejo de estudiar estructuras tridimensionales bajo los conceptos antes mencionados utilizando el criterio de [7] debido a la cantidad de elementos tipo armadura que se generarían al discretizar los MCR; esto generaría un gran esfuerzo computacional y numérico, pero, sin duda alguna, los resultados que se obtendrían serían adecuados al comportamiento no lineal esperado de las estructuras con MCR ante excitaciones sísmicas de baja a gran intensidad.

\section{Financiamiento}

Artículo de investigación científica derivado del proyecto de investigación "Modelaciones analíticas de muros de concreto reforzado" desarrollado con el apoyo del Grupo de Investigación en Construcción, Geotecnia y Medio ambiente, GIGMA, de la Universidad Francisco de Paula Santander Ocaña y el Instituto de Ingeniería de la Universidad Autónoma de México. Fecha de inicio: enero de 2017; fecha de finalización: diciembre de 2017.

\section{Agradecimientos}

Agradecimiento especial al Instituto de Ingeniería de la UNAM por ser parte muy importante en la realización de este estudio.

\section{REFERENCIAS}

[1] J.C. Vielma, A.H. Barbat y S. Oller, "Respuesta no lineal de edificios de concreto armado diseñados para baja ductilidad," Boletín Técnico, vol. 45, no. 2, pp. 12-34, jul., 2007. [Online]. Disponible en: http://www.scielo.org.ve/scielo. php?script=sci_arttext\&pid=S0376-723X2007000200002 \&lng=es\&tlng=es.

[2] B. Wang, H. Jiang y X. Lu, "Investigaciones experimentales y numéricas sobre el comportamiento sísmico de muros de núcleo de hormigón armado de armadura de acero", Eng. Struct., vol. 140, pp. 164-176, jun., 2017. https://doi. org/10.1016/j.engstruct.2017.02.055

[3] D. Bravo, R. Rincón y G. Gutiérrez, "Evaluación del uso de muros estructurales de corte como elementos sismorresistentes en edificaciones aporticadas de concreto armado," Revista Estudiantil URU, no. 1, pp. 11-20, ene., 2015. 
[4] P. Sengupta y B. Li, "Hysteresis Behavior of Reinforced Concrete Walls", J. Struct. Eng., vol. 140, no. 7, jul., 2014. https://doi.org/10.1061/(ASCE)ST.1943-541X.0000927

[5] J. I. S. de la Cruz y A. J. Pino, "Rigidez y flexibilidad en el análisis de diafragmas flexibles," Maskana, pp. 1-11, 2014.

[6] F. De-cheng, R. Xiao-Dan y L. Ren, "Cyclic behavior modeling of reinforced concrete shear walls based on softened damage-plasticity model", Eng. Struct., vol. 166, pp. 363-375, mar., 2018. https://doi.org/10.1016/j.engstruct.2018.03.085

[7] M. Panagiotou, J. I. Restrepo, M. Schoettler y G. Kim, "Nonlinear cyclic truss model for reinforced concrete walls," ACI Structural Journal, vol. 109, no. 2, pp. 205214, mar., 2012.

[8] P. Kotronis, J. Mazars, X. H. Nguyen, I. Nicolas, J-M. Reynouard y P. Bisch, "The seismic behavior of reinforced concrete structural walls: experiments and modeling," The 1755 Lisbon Earthquake: Revisited, Springer, Netherlands, 363-376, ene., 2009.

[9] S. A. Williams, "Numerical Analysis of Reinforced Masonry Shear Walls Using the Nonlinear Truss Approach", PhD. Dissertation, Virginia Tech, Blacksburg, VA, 2014.

[10] P. G. Papadopoulos, H. Xenidis, P. Lazaridis, A. Diamantopoulos, P. Lambrou y Y. Arethas, "Achievements of truss models for reinforced concrete structures," OJCE, vol. 2, no. 3, pp. 125-131, sep. 2012. http://dx.doi. org/10.4236/ojce.2012.23018

[11] J. M. Vallenas, V. V.Bertero y E. P. Popov, "Hysteretic behaviour of reinforced concrete structural Mwalls," Report No. UBC/EERC-79/20, University of California, Berkeley, 1979.

[12] H. Jiang y B. Fu., "Nonlinear Cyclic Truss Model for Reinforced Concrete Walls Discussion," ASJ, vol. 110, no. 1, pp. 155, ene., 2013.

[13] C. Celi, J. Pantoja, C. Ayala y D. Sosa, Capacity Curves of Structural Typologies of Quito - Ecuador, Project GEM - SARA, jul. 2016. [Online]. Available: https:// www.researchgate.net/publication/305654264

[14] F. Mazzoni, M. McKenna, M. Scott y G. Fenves, "Open system for earthquake engineering simulation, user command-language manual", Report NEES grid-TR 200421. PEER, University of California, Berkeley, CA, 2004. Disponible en: http://opensees.berkeley.edu

[15] C. Ayala y J.L. Almazán, "Modelo celosía no-lineal para muros de albañilería armada de bloque con relleno parcial", en XI CSIS, 2015, https://doi.org/10.13140/ RG.2.1.2985.2885

[16] J. Hoshikuma, K. Kawashima, K. Nagaya y A. W. Taylor, "Stress-strain model for confined reinforced concrete in bridge piers," Journal of Structural Engineering, vol. 123, no. 5, pp. 624-633, 1997.

[17] N. J. Stevens, S. M. Uzumeri, M. P. Collins y T. G. Will, "Constitutive model for reinforced concrete finite element analysis," ACI Structural Journal, vol. 88, no. 1, pp. 49-59, 1991.

[18] A. Giuffrè y E. Pinto, "Il comportamento del cemento armato per sollecitazioni cicliche di forte intensità", Giornale del Genio Civile, mayo, 1970.

[19] F. C. Filippou, E. P. Popov y V. V. Bertero, "Effects of Bond Deterioration on Hysteretic Behavior of Reinforced Concrete Joints", EERC, Report UCB/EERC-83/19, pp. 83-19, University of California, Berkeley, mayo, 1983

[20] H. Jiang y B. Fu, "Nonlinear Cyclic Truss Model for Reinforced Concrete Walls Discussion," Aci Struc. J., vol. 110, no. 1, p. 155, feb., 2013.

[21] M. H. Scott, "Numerical Integration Options for the Force-Based Beam-Column Element in OpenSees," Force-Based Element Integration Options in OpenSees, ene, 2011. [Online]. Available:http://opensees.berkeley.edu/ wiki/images/a/ab/IntegrationTypes.pdf
Alberto Vásquez Martínez obtuvo el título de ingeniero civil en el Instituto Tecnológico de Villahermosa, México, además, se recibió con mención honorifica como maestro en ingeniería estructural por parte de la Universidad Nacional Autónoma de México. Ha impartido diversas materias de ingeniería civil a nivel de licenciatura y posgrado en la Universidad Autónoma de Guadalajara, Campus Tabasco. Actualmente es candidato a doctor en ingeniería sísmica en la Universidad Nacional Autónoma de México. Sus líneas de investigación son: confiabilidad y vulnerabilidad sísmica en estructuras de concreto reforzado y mampostería. Asimismo, es consultor independiente en diseño estructural de diversos tipos de estructuras. https://orcid. org/0000-0003-4909-6501

Romel Jesús Gallardo Amaya recibió el título de ingeniero civil en 1998 y de magíster en geotecnia en 2014 de la Universidad Industrial de Santander (Bucaramanga, Colombia). Vinculado desde el 2009 como docente de tiempo completo y director del grupo de investigación GIGMA en la Facultad de Ingeniería de la Universidad Francisco de Paula Santander, Ocaña. Sus intereses de investigación incluyen gestión del riesgo, métodos geofísicos, interacción suelo-estructura y mejoramiento de suelos. https://orcid. org/0000-0002-4740-4841 